Internist 2013 $\cdot 54: 909-910$

DOI 10.1007/s00108-012-3138-y

Online publiziert: 3. Juli 2013

๑) Springer-Verlag Berlin Heidelberg 2013
M. Battegay ${ }^{1}$ G. Fätkenheuer ${ }^{2}$ 'W.V. Kern ${ }^{3}$

${ }^{1}$ Klinik für Infektiologie \& Spitalhygiene, Universitätsspital Basel

${ }^{2}$ Klinik 1 für Innere Medizin, Uniklinik Köln

${ }^{3}$ Zentrum Infektiologie und Reisemedizin, Medizinische Klinik, Universitätsklinikum Freiburg

\section{Klinische Infektiologie}

\section{Interdisziplinär und einzigartig}

In den letzten 10 Jahren widmete sich DerInternist in insgesamt 10 Schwerpunktheften infektiologischen Themen - von der Reisemedizin über Parasitosen, Impfungen und Infektionsprävention bis zu spezifischen Themen wie Lungeninfektionen oder der HIV-Infektion. In Lehrbüchern der Inneren Medizin ist das Kapitel „Infektionskrankheiten“ meist das umfassendste. Dies hat mehrere Gründe: zum einen die Vielfalt der Erreger, die Viren, Bakterien, Pilze sowie Protozoen und Parasiten einschließt, zum anderen die Palette der Pathogen-Wirt-Interaktionen mit einer zunehmenden Zahl immunsupprimierter Patienten, aber auch mit unterschiedlichen Verläufen bei Kleinkindern, Erwachsenen und betagten Patienten. Hinzu kommen spezifische Besonderheiten von Infektionskrankheiten, die sie von anderen Krankheitsentitäten unterscheiden und einzigartig machen. $\mathrm{Zu}$ diesen gehören u. a.

- die Übertragbarkeit mit dem daraus resultierenden Potenzial der Entstehung von Epidemien oder Pandemien;

- die Bildung einer stabilen Immunität gegen manche Infektionen;

- das Anpassungspotenzial von Mikroorganismen und die Entstehung neuer Erreger oder von Erregern mit veränderten Eigenschaften;

- die Möglichkeit einer effektiven Prävention oder sogar Eradikation.

Insbesondere präventive Maßnahmen diese schließen nicht nur Impfungen ein - haben eine herausragende medizinische und gesellschaftliche Bedeutung.
Das Auftreten von Infektionskrankheiten in den unterschiedlichsten Organen und damit ihre Zuordnung zu den verschiedenen Disziplinen sowohl der Inneren als auch der gesamten Medizin machen die Infektiologie zu einem Querschnittsfach, das originär auf eine gute interdisziplinäre Zusammenarbeit ausgerichtet ist. Angesichts der zunehmenden Spezialisierung und fachlichen Aufsplitterung der Medizin stellt die Infektiologie mit ihren Problemstellungen (z. B. der Abklärung von Fieber, importierten Erkrankungen, opportunistischen Infektionen und Epidemien) und ihren Verzweigungen in alle Teilgebiete der Inneren Medizin heute einen Kernbereich dieses Faches dar. In diesem Sinne möge der aktuelle Schwerpunkt wie die vorausgegangenen Schwerpunkte dazu beitragen, das spezielle klinische Wissen - dies gilt selbstverständlich analog für andere Fachdisziplinen - sowie die Interdisziplinarität hervorzuheben.

》) Die Infektiologie ist originär auf eine gute interdisziplinäre Zusammenarbeit ausgerichtet

Weltweit, insbesondere aber in den meisten Ländern Europas und in Amerika, haben sich in den letzten Jahrzehnten klinische Abteilungen für Infektiologie etabliert; vielfach schließen diese auch die Krankenhaushygiene ein. Diese Entwicklung erfolgte aus der Einschätzung heraus, dass für eine qualifizierte infektiologische Versorgung eine spezielle Expertise erforderlich ist, die eine entsprechende Weiterbildung voraussetzt. Die aktuellen und die voraussehbaren Probleme, die Infektionskrankheiten bereiten, machen eine Stärkung der Infektiologie in Deutschland als Weiterbildungsfach der Inneren Medizin notwendig. Für junge Kollegen in der Weiterbildung stellt die Infektiologie mit ihrem breiten Spektrum an Krankheitsbildern, ihrem Bezug zu allen Teilbereichen der Medizin und ihrem interdisziplinären Vorgehen ein attraktives Gebiet dar.

In den vorliegenden Schwerpunktbeiträgen zeigt sich exemplarisch die Bedeutung der interdisziplinären Zusammenarbeit bei infektiologischen Themen, aber auch die Notwendigkeit spezieller Kenntnisse und Expertise. Im ersten Artikel beschreiben A. Schibli et al. die initiale Beurteilung und erste Abklärungsschritte bei Patienten mit Infektionskrankheiten, insbesondere bei einem Status febrilis. Eine lebensbedrohliche Erkrankung muss schnell erkannt, eine empirische Antibiotikatherapie rasch initiiert werden. Gerade in diesen Situationen ist die Abnahme von Blutkulturen und sonstigen Proben für die Erregerdiagnostik vor Beginn der Antibiotikatherapie unverzichtbar, um die mikrobiologische Diagnose zu sichern.

In einem weiteren Artikel stellen B. Salzberger et al. die Ursachen und das Management schwerer Infektionen vor, besonderes Gewicht wird auf die Sepsis gelegt. Deren klinische Definition wird eingehend beschrieben. Zudem werden wichtige zur Sepsis führende Infektionskrankheiten dargestellt, z. B. die Pneumonie, schwere Weichteilinfektionen, die Meningitis oder die Endokarditis.

Weltweit nehmen resistente Erreger aufgrund des zu hohen Antibiotikakon- 
sums durch Mensch und Tier zu. Namentlich in den letzten Jahren kam es zur Ausbreitung hochresistenter gramnegativer, teilweise sogar carbapenemresistenter Bakterien. In der Folge sind viele Breitspektrumantibiotika nicht mehr oder nur noch teilweise wirksam. H. Stocker $u$. W.V. Kern stellen mit Colistin eine Subs$\operatorname{tanz}$ vor, die als eines von mehreren älteren Antibiotika mit Wirkung gegen multiresistente gramnegative Bakterien derzeit eine Renaissance erlebt bzw. neu bewertet wird. Der Artikel beschreibt den Stellenwert von Colistin bei schweren Infektionen (häufig in Kombination mit anderen Substanzen), fasst neue Daten zur Substanz zusammen und gibt wichtige Hinweise für den praktischen Einsatz.

Die Interdisziplinarität der Infektiologie zeigt sich im Artikel von N. Jung et al. über die Spondylodiszitis. Diese Infektion gehört zwar nicht zu den häufigsten Erkrankungen, ist jedoch ein sehr gutes Beispiel für spezifische infektiologische Algorithmen, die auf dem Wissen über verursachende Erreger, diagnostische Probleme sowie zielgerichtete Therapien basieren. Vielfach ist hier ein interdisziplinäreres Vorgehen nötig, so beispielsweise unter Einbeziehung von Kollegen der Radiologie, Chirurgie oder Orthopädie für eine computertomographisch gesteuerte oder offene Biopsie zur Erregersicherung.

S. Rosseau, H. Schütte u. N. Suttorp beschreiben die ventilatorassoziierte Pneumonie als diagnostische und therapeutische Herausforderung. So muss etwa die Wertigkeit der mikrobiologischen Untersuchung eines Trachealsekrets bei beatmeten Patienten eingeschätzt und damit überlegt werden, ob eine Antibiotikatherapie eingeleitet oder verändert werden sollte. Diese Entscheidungen sind nicht einfach, da die Bedeutung eines Erregernachweises häufig nicht selbst evident ist und da eine ,automatische“ Änderung der Antibiotikatherapie - in der Regel in Richtung einer Verbreiterung des Spektrums - die Zunahme von Resistenzen begünstigt. Der Artikel bietet bei solchen Entscheidungen Unterstützung.

Wir hoffen, Ihnen mit diesem Schwerpunkt interessante und wichtige Einblicke in die klinische Infektiologie zu geben und die Bedeutung dieses Fachs in der Inneren Medizin darstellen zu können. Zahlreiche fachliche Anregungen und viel Freude bei der Lektüre wünschen Ihnen

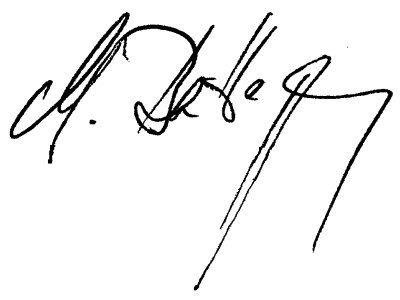

M. Battegay

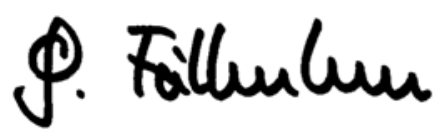

G. Fätkenheuer

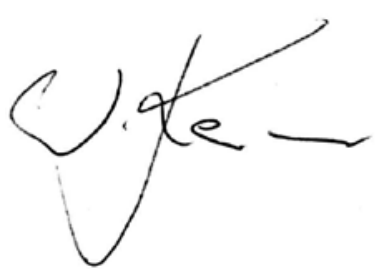

W.V. Kern

\section{Korrespondenzadresse}

Prof. Dr. M. Battegay

Klinik für Infektiologie \& Spitalhygiene,

Universitätsspital Basel

Petersgraben 4, 4031 Basel

Schweiz

manuel.battegay@usb.ch

\section{Ärzte Zeitung digital ist} Fachmedium des Jahres

Digitale Formate sind auch in der medizinischen Fachpresse im Trend, und Springer Medizin setzt mit seinen Angeboten Maßstäbe. Die Fachverlagsgruppe hat für „Ärzte Zeitung digital", die App-Ausgabe der Tageszeitung, den renommierten Preis „Fachmedium des Jahres" 2013 in der Kategorie „Bestes Mobiles Angebot" erhalten. Der Preis wird von der Deutschen Fachpresse in mehreren Branchen- und Sachkategorien verliehen. Die Deutsche Fachpresse ist die Marketing- und Dienstleistungsplattform für alle Anbieter von Fachinformationen im beruflichen Umfeld.

Mit dem Preis werden Publikationen gewürdigt, „'die beispielhaft für die vielen hochwertigen gedruckten und digitalen Informationsangebote aus Fachmedienhäusern in Deutschland stehen".

"Wir haben mit der „Ärzte Zeitung digital" konsequent unsere Tageszeitung für Ärzte in das digitale Zeitalter überführt", kommentiert Harm van Maanen, Executive Vice President von Springer Medizin, die Auszeichnung für die Fachtageszeitung. Die Strategie der Digitalisierung werde konsequent weiter verfolgt, so van Maanen weiter. So soll die App-Ausgabe, die bisher weitgehend die Inhalte der gedruckten Tageszeitung abbildet, eigenständig werden.

Die Ausgabe der „Ärzte Zeitung“ fürs iPad ist erstmals im November 2012 erschienen - im September 2013 folgt die Version für Android-Tablets. Das Angebot vervollständigt die digitalen Formate der Zeitung, die damit online über aerztezeitung.de, auf Smartphones (News App fürs iPhone sowie für Smartphones optimierte Website) und eben auch mit einer eigenen Ausgabe für Tablets erreichbar ist.

Quelle: Ärzte Zeitung 